\title{
Relating illness complexity to reimbursement in CKD patients
}

\author{
Russell W Bessette' \\ Randy L Carter ${ }^{2,3}$ \\ 'Department of Health Sciences, \\ Institute for Healthcare Informatics, \\ ${ }^{2}$ Department of Biostatistics, \\ ${ }^{3}$ Population Health Observatory, \\ University at Buffalo, State University \\ of New York, Buffalo, NY, USA
}

This article was published in the following Dove Press journal: International Journal of Nephrology and Renovascular Disease 16 September 2011 Number of times this article has been viewed

Background: Despite significant investments of federal and state dollars to transition patient medical records to an all-electronic system, a chasm still exists between health care quality and payment for it. A major reason for this gap is the difficulty in evaluating health care outcomes based on claims data. Since both payers and patients may not appreciate how illness complexity impacts treatment outcomes, it is difficult to determine fair provider compensation.

Objectives: Chronic kidney disease (CKD) typifies these problems and is often associated with comorbidities that impact cost, health, and work productivity. Thus, the objective of this study was to evaluate an illness complexity score (ICS) based on a linear regression of select blood values that might assist in predicting average monthly reimbursements in CKD patients. A second objective was to compare the results of this ICS prediction to results obtained by prediction of average monthly reimbursement using CKD stage. A third objective was to analyze the relationship between the change in ICS, estimated glomerular filtration rate (eGFR), and CKD stage over time to average monthly reimbursement.

Methods: We calculated parsimonious values for select variables associated with CKD patients and compared the ICS to ordinal staging of renal disease. Data from 177 de-identified patients over 13 months was collected, which included 15 blood chemistry observations along with complete claims data for all medical expenses. To test for the relationship between average blood chemistry values, stages of $\mathrm{CKD}$, age, and average monthly reimbursement, we modeled an association through a linear regression function of age, eGFR, and the Z-scores calculated from average monthly values of phosphorus, parathyroid hormone, glucose, hemoglobin, bicarbonate, albumin, creatinine, blood urea nitrogen, potassium, calcium, sodium, alkaline phosphatase, alanine aminotransferase, and white blood cells.

Results: The results of our study demonstrated that the association between average ICS values throughout the entire study period predicted average monthly reimbursements with an $R^{2}$ value of 0.41. Comparing that value to the association between the average CKD stage and average monthly reimbursement demonstrated an $R^{2}$ value of 0.08 . Thus, ICS offers five times greater sensitivity over CKD staging as a measure of illness complexity.

Conclusion: Sorting the patient population by changes in CKD stage or ICS over the entire study period revealed significant differences between the two scoring methods. Groups scored by ICS demonstrated greater sensitivity by capturing dysfunction in other organ systems and had a better association with reimbursement than groups scored by CKD staging.

Keywords: chronic kidney disease, illness complexity score, reimbursement, disease severity

\section{Introduction}

Many policy makers have suggested that "quality of health care can be precisely defined and measured with a degree of scientific accuracy comparable to most measures 
used in clinical practice."1 In 1994, the Institute of Medicine supported that view and added that "quality of care is the degree to which health services for individuals and populations increase the likelihood of desired health outcomes and are consistent with current professional knowledge."

However despite these expectations, along with federal and state investments to transition patient medical records to an all-electronic system, a chasm still exists between reporting health care quality and measuring payment for it. Petersen et al, in an extensive review of the literature, compared various methods to improve quality through pay-for-performance programs. ${ }^{3}$ Their analysis concluded that most financial incentives were focused on the delivery of prevention services rather than health outcomes. Other investigators reported that so-called pay-for-performance programs impact some patients negatively, particularly those with mental illness and chemical dependency. ${ }^{4-6}$ These conclusions support the analysis of Porter and Teisberg and others that American health care competes on delivery of the lowest procedure price rather than a value-based outcome for individual patients. ${ }^{7-9}$ It is this enigma of value vs price that challenges American health care. Determining value requires an appreciation of the relationship between illness severity, expected outcome, and cost.

Currently most payers address high cost and outcome by encouraging the delivery of preventive medical services, such as up-to-date immunizations, early diagnostic studies including mammography, colonoscopy, Pap smears, prostatespecific-antigen testing, or education in healthy life styles. ${ }^{10,11}$ Though these services are valuable, patients still develop chronic illnesses that require treatment or palliative care. Indeed, such conditions dominate health care budgets. In order to compensate providers fairly, it would be helpful to understand the level of illness severity in each patient prior to starting treatment. In this manner, both payers and providers can evaluate cost and treatment outcome among patients of comparable complexity.

Chronic kidney disease (CKD), which has five stages of severity based on a declining glomerular filtration rate (GFR), is typically evaluated and compensated according to disease stage. Yet patients within any single stage may demonstrate higher complexity due to comorbid factors such as hypertension, diabetes, osteopenia, and congestive heart failure among others. Unfortunately without a scale to grade illness complexity in individual patients, payers must rely on disease stage or capitation agreements based on assumed group complexity to aggregate cost and compensate providers. ${ }^{12,13}$ Though pay-for-performance programs attempt to improve both care and cost, if illness complexity before treatment is unknown, then value-based outcomes also remain unknown. ${ }^{14-16}$

With the introduction of accountable care organizations in the United States, there is renewed focus to link provider compensation to quality outcomes through risk adjusted capitation agreements within a patient centered medical home. However, determining risk in populations challenged by social and geographic barriers is difficult and often compels providers to accept reimbursement based on healthier populations. The end result is generally lower care for underserved populations.

In a previous study, the authors reported on patients with CKD, many with multiple comorbidities, and demonstrated a relationship between blood chemistry values and high-cost hospitalization. ${ }^{17}$ In this study, an example population of CKD patients with multiple comorbid conditions was analyzed in order to explore the association between an illness complexity score (ICS) based on routine blood chemistry values to reimbursement.

The objective of this study was to formulate an ICS based on a linear regression of select blood values that could predict average monthly reimbursements in CKD patients. A second objective was to analyze the association between reimbursement and CKD stage. A third objective was to compare performance between these two methods for predicting average monthly reimbursement.

\section{Method}

\section{Samples analyzed}

The data set analyzed included 1104 de-identified patients from a local managed care organization's kidney disease registry who had received treatment from November 2007 through November 2008. Patients without a calculated stage of kidney disease or a repeated estimated GFR (eGFR) that was at or below $60 \mathrm{~mL} /$ minute over a 3-month period were excluded (216 patients), since they may have represented acute renal disease, which was not the focus of this study. After exclusion, $888 \mathrm{CKD}$ patients remained in the sample.

\section{Variable definitions}

A total of 18 blood tests were requested from the managed care organization for analysis by a consulting group of university nephrologists. The choice of tests was made based on each variable's perceived importance in monitoring the health of CKD patients. The 18 blood tests were: serum phosphorus, parathyroid hormone, glucose, glycolated 
hemoglobin, hemoglobin, hematocrit, bicarbonate, albumin, creatinine, blood urea nitrogen, potassium, calcium, sodium, alkaline phosphatase, alanine aminotransferase, bilirubin, white blood cells (leukocytes), and eGFR. The data set also included the complete financial profile for all medical claims that were paid for services for these patients over the same time period. These costs were also studied.

Since blood tests ordered by physicians showed marked variation in selection and repetition, the remaining pool of 888 patients were filtered into a data set of 177 patients with no missing values for the following 15 tests that were repeated at least twice or more over the study period: phosphorus, parathyroid hormone, glucose, hemoglobin, bicarbonate, albumin, creatinine, blood urea nitrogen, potassium, calcium, sodium, alkaline phosphatase, alanine aminotransferase, white blood cells, and eGFR. The blood tests for all patients at all times were performed by the same laboratory. Thus, the units of measurement and normal range for each test were common to all observations.

Data for each patient was organized on a spreadsheet with columns labeled for patient identification, date of medical service, payments for all reimbursed medical services, CKD stage, and results of each blood test. Rows were grouped by patient identification and chronological dates for medical services. Since all 15 blood tests were not repeated on each date that a medical procedure was delivered, test results were carried forward to subsequent rows until replaced by a fresh test result. The average number of data rows for each patient was 13.1 with most patients having one or more tests repeated in eight of the 13 study period months.

Next, with the exception of age and eGFR, each blood test result was converted to a Z-score as follows: the midpoint of the normal range for each test was taken as the mean, and the range divided by four as the standard deviation for a nondiseased normal population. Each lab test was standardized using this mean and standard deviation to obtain a Z-score for each variable for each patient. Next the Z-scores for each patient's test results, along with their age, eGFR, and all reimbursements in each respective column were averaged by month.

A summary spreadsheet contained 177 lines for each patient's average age, eGFR, average monthly reimbursement, and average $\mathrm{Z}$-scores for all tests over the entire study period. These averaged values for all variables were utilized in a linear regression equation to develop a predictor for average monthly reimbursement in each patient. Graphs and significance levels were calculated on these results. The same regression coefficients used in the preceding equation were also employed to calculate ICS for each patient on each date of service in order to analyze change.

The change in ICS from start to end of the study period was used to cohort the population into three outcome groups: better, same, or worse. Changes in CKD stage from beginning to end of the study period was calculated directly from the laboratory values at date of service.

\section{Statistical methods}

To test for the relationship between average blood chemistry values, stages of CKD, age, and average monthly reimbursement, we modeled that association through a linear regression function of age, eGFR, and the Z-scores calculated from average monthly values of phosphorus, parathyroid hormone, glucose, hemoglobin, bicarbonate, albumin, creatinine, blood urea nitrogen, potassium, calcium, sodium, alkaline phosphatase, alanine aminotransferase, and white blood cells. A backward selection strategy was then employed to derive a parsimonious model containing only significant predictors. At each step, the explanatory variable with the highest $P$ value greater than 0.10 was deleted. If its deletion resulted in another variable that had been significant $(P<0.10)$ previously becoming nonsignificant, then the deleted variable was added back into the model and the variable with the next largest $P$ value greater than 0.10 was deleted. These steps were repeated until only significant variables $(P<0.10)$ remained in the model.

These analyses produced a regression table for the final model with an estimated intercept and regression coefficients for each explanatory variable, along with calculated $P$ values. Next employing the regression coefficients calculated for the most parsimonious variables, these coefficients were employed in a regression equation to calculate a linear predictor by multiplying each appropriate regression coefficient with their respective averaged explanatory variable and summed. The results for each patient were plotted on a scatter plot of ICS vs the natural logarithm for each patient's average monthly reimbursement.

Next, employing the regression coefficients calculated for the most parsimonious variables, an ICS was calculated for each patient on each date of service with no missing variables. As described previously, the regression coefficients used to calculate each ICS on each date of service for each patient were derived from the linear regression calculation for the entire population based on average $\mathrm{Z}$-scores for each patient. The chronological change calculated in this way throughout the study period permitted analysis of the relationship of outcome result (ie, change in ICS) to reimbursement. 
Next, the coefficients of the linear regression of the average natural logarithm for monthly reimbursements on average CKD stage categories for each patient over the entire study period were estimated. In a manner similar to developing a simple linear predictor for multiple blood tests above, the regression coefficient for CKD stage was multiplied with each observed indicator variable for stage and summed with the estimated intercept to produce a predicted value of reimbursement based on stage. Subsequently, these values were plotted in a scatter plot against the average natural logarithm for monthly reimbursement.

Finally, in order to evaluate the relationship between outcome and reimbursement, the study pool was sorted by change in ICS and CKD stage from first to last observation month. Since ICS is a continuous variable and stage is ordinal with a wide range for each stage, the change in eGFR from first to last observation month was also evaluated. Since patients scored by any measure could remain the same, improved, or worse, the pool was divided into three groups based on ending CKD stage, ending ICS, and ending eGFR. The average values for each patient's starting and ending CKD stage, ICS, or eGFR were evaluated by a paired $t$-test, and the significance for the change in average reimbursement within each subset was evaluated by an ANOVA calculation. In order to illustrate the predictive power of ICS and CKD staging to average monthly reimbursement, the average ICS and CKD stages from start to end of the study period for each patient were plotted in line graphs and compared to a similar plot for the log of average payments. In addition, $R^{2}$ values were calculated from the simple linear regressions.

\section{Results}

Table 1 displays the coefficients and $P$ values from the regression of the average logarithm of monthly reimbursement on the full set of variables in the table. This regression was based on the sample of 177 patients with observations on all variables analyzed. The overall $R^{2}$ value from the regression was $0.424(P=0.0005)$.

Although the overall $P$ value for the association of these variables to average monthly cost was significant, as shown in Table 1 , a number of variables had $P$ values that were not significant. After a step-wise elimination of the least significant variable at each step, a parsimonious model that did not suffer from multicollinearity was obtained and is presented in Table 2. This parsimonious set of variables had an overall $P$ value of 0.0005 , with an $R^{2}$ of 0.41 and an adjusted $R^{2}$ of 0.37 .
Table I Calculated coefficients and $P$ values from the regression of the average logarithm of monthly reimbursement for the variables listed in the first column

\begin{tabular}{lll}
\hline & Variable coefficient & $\boldsymbol{P}$ value \\
\hline Constant & -2.84 & \\
Age & 0.01 & 0.10 \\
Stage CKD & 1.24 & 0.03 \\
PO4 & 0.15 & 0.04 \\
PTH & 0.00 & 0.33 \\
Glucose & 0.01 & 0.57 \\
Hemoglobin & -0.33 & 0.00 \\
Bicarbonate & -0.03 & 0.71 \\
Albumin & -0.28 & 0.00 \\
Creatinine & 0.03 & 0.01 \\
BUN & 0.01 & 0.55 \\
Potassium & -0.07 & 0.33 \\
Calcium & 0.08 & 0.25 \\
Sodium & 0.06 & 0.53 \\
Alk-P & -0.01 & 0.92 \\
ALT & 0.31 & 0.00 \\
WBC & 0.17 & 0.00 \\
eGFR & 0.08 & 0.00 \\
\hline
\end{tabular}

Abbreviations: Alk-P, alkaline phosphatase; ALT, alanine aminotransferase; BUN, blood urine nitrogen; CKD, chronic kidney disease; eGFR, estimated glomerular filtration rate; $\mathrm{PO}$, phosphate; $\mathrm{PTH}$, parathyroid hormone; WBC, white blood cells.

The association between the ICS derived from this model and the average logarithm for monthly reimbursement for all health care services for each patient is shown in the scatter plot of Figure 1. The average ICS over the entire study period are displayed on the $\mathrm{x}$-axis, and are derived from the intercept plus a linear predictor derived by the sum of the Z-scores for each test multiplied by its respective variable coefficient shown in Table 2. That is, the ICS was defined as the predicted value of the average logarithm of reimbursement. The average logarithms for monthly reimbursements for all health care services are displayed on the y-axis.

Table 2 Calculated variable coefficients and $P$ values for the parsimonious model obtained after a step-wise elimination of the least significant variables previously shown in Table I

\begin{tabular}{lll}
\hline & Variable coefficient & $P$ value \\
\hline Constant & -2.86 & \\
Age & 0.01 & 0.09 \\
CKD Stage & 1.31 & 0.02 \\
PO4 & 0.17 & 0.01 \\
Hemoglobin & -0.31 & 0.00 \\
Albumin & -0.25 & 0.00 \\
Creatinine & 0.03 & 0.00 \\
ALT & 0.30 & 0.00 \\
WBC & 0.16 & 0.00 \\
eGFR & 0.08 & 0.00 \\
\hline
\end{tabular}

Abbreviations: ALT, alanine aminotransferase; CKD, chronic kidney disease; eGFR, estimated glomerular filtration rate; WBC, white blood cells. 


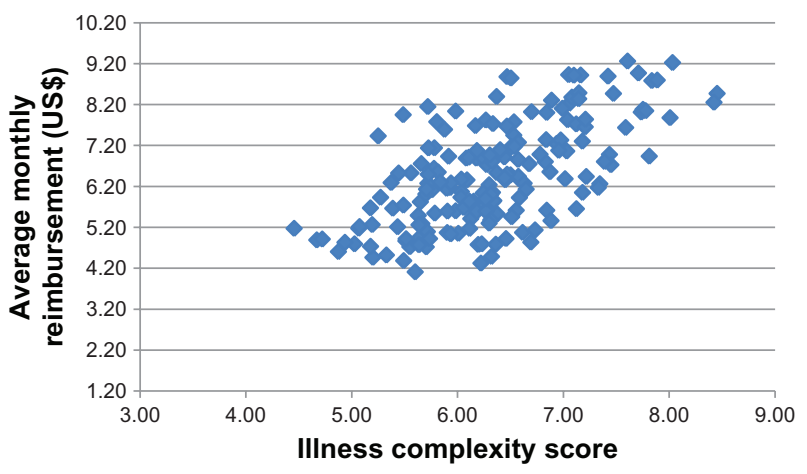

Figure I Scatter plot of illness complexity score (x-axis) derived as the predicted value from the linear regression for age, chronic kidney disease stage, serum phosphorus, hemoglobin, albumin, creatinine, alanine aminotransferase, white blood cells, and estimated glomerular filtration rate vs the natural logarithm of average monthly reimbursement (y-axis) for total health care services in 177 chronic kidney disease patients over I year.

As shown in Figure 1, complexity scores ranged from 4.45 to 8.45 (x-axis) and were associated with increasing average monthly reimbursements: 4.11 to 9.26 (US\$61 to US\$10,509; y-axis). The $R^{2}$ value for the relationship between ICS and the average natural logarithm for monthly reimbursement for all health care services is 0.41 .

Figure 2 in contrast is the scatter plot for the same CKD population, but sorted by average CKD stage for each patient over the study period. The average values for CKD stages are based on a calculated eGFR (Modification of Diet in Renal Disease 4) and weighted by their regression coefficients which are displayed on the $\mathrm{x}$-axis, while the average natural logarithm for monthly reimbursement for all health care services is shown on the y-axis.

The variation in average monthly dollars for all four CKD stages shown in Figure 2 varies from 4.11 to 9.26

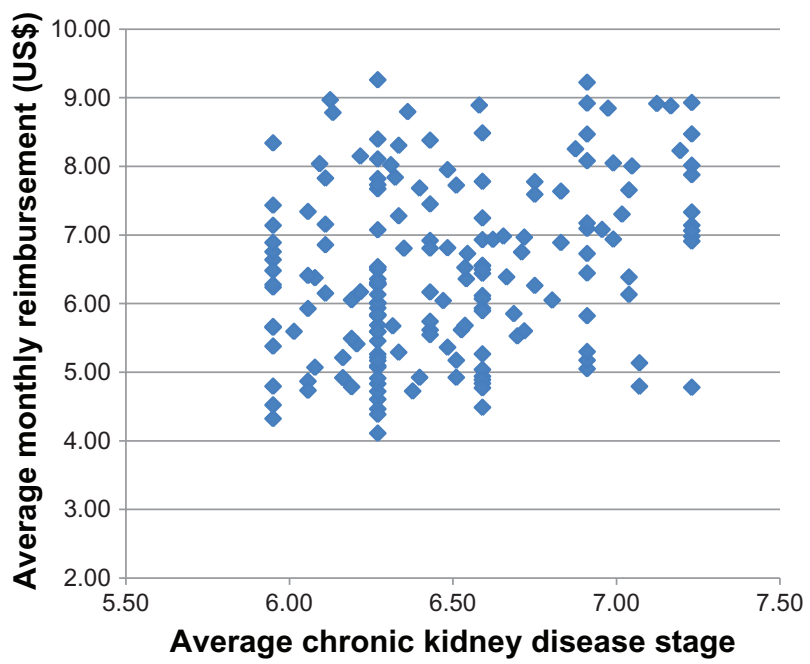

Figure 2 Scatter plot of the linear predictor for average chronic kidney disease stage (x-axis) vs the natural logarithm of average monthly reimbursement ( $y$-axis) for all health care services in 177 patients.
(US\$61 to US\$10,509). Interestingly, the widest range of reimbursements was seen in the vertically aggregated diamonds seen at $\mathrm{x}$-axis $=6.27$ which is associated with $\mathrm{CKD}$ stage 3B. The linear regression for the association between average stage of CKD and average monthly reimbursement had an $R^{2}$ value of 0.083 with an adjusted $R^{2}$ of 0.078 .

In order to compare the performance between average ICS and average CKD stage at predicting average natural logarithm for monthly reimbursement in each patient, the two patient pools were rank ordered by reimbursement amount from smallest to largest and plotted by line graphs as shown in Figures 3 and 4. Figure 3 is the line graph for 177 patients displaying the relationship between ICS and the average natural logarithm for total monthly reimbursements. The irregular red line depicts ICS values (y-axis) for each patient displayed on the $\mathrm{x}$-axis. The green slightly sigmoidshaped line illustrates the values for the natural logarithm of average monthly reimbursements for each patient (also on the y-axis scale). The range of these scores was from an ICS value of 5.6 to 7.7. As suggested by the $R^{2}$ value of 0.41 , there is correlation of the predicted ICS values to average monthly reimbursement in the midrange of the line graphs with a symmetrical divergence of ICS values at both the upper and lower regions of the graph.

In contrast, Figure 4 demonstrates a line graph comparing average CKD stage to the average natural logarithm for monthly reimbursements in 177 patients. The linear trend line for the beta-weighted average stages of CKD ranged from a value of 6.2 to 6.9 with an $R^{2}$ value of 0.083 . The small correlation of weighted CKD stage values with the line plot for average reimbursement confirms the predicted relationship by a wide divergence of the stage predicted values from the average monthly reimbursements.

In order to evaluate changes observed in ICS and CKD stage over the entire study period and to correlate those changes to reimbursement, the patient pool was sorted by change in both ICS and CKD stage from first to last observation and compared to their respective average monthly reimbursements. Since ICS is a continuous variable, while CKD stage is ordinal with a wide range of eGFR values in each stage, the patient pool was also sorted by change in eGFR values from start to end. The results of this pool segregation are displayed in Table 3 which lists the natural logarithm values for average monthly reimbursement for those patients who ended the study period with the same, better, or worse ending eGFR, ICS, or CKD stage. The first full row in Table 3 displays the number of patients with the same, better, or worse ending values for each scoring method. 


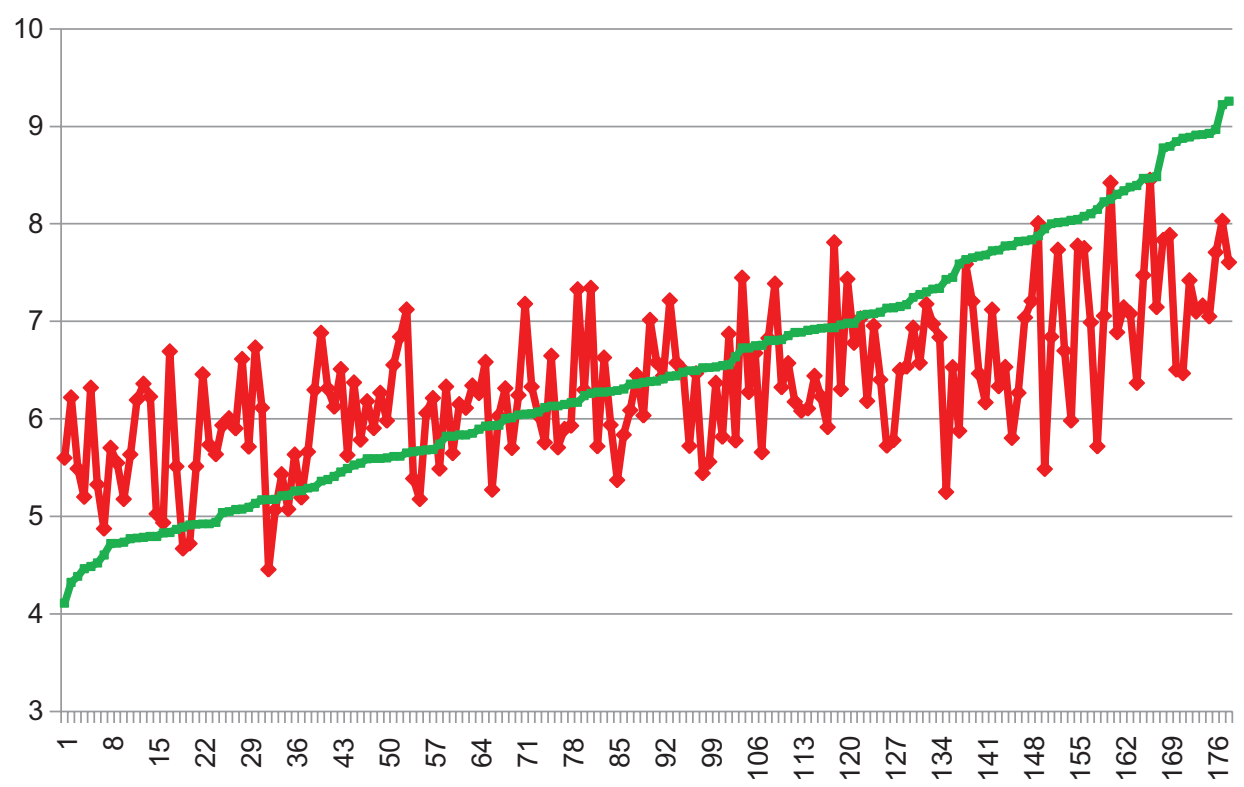

Figure 3 Line graph for 177 renal patients (x-axis) depicting each patient's average illness complexity score (red line) along with each patient's respective natural logarithm for average monthly reimbursement (green line). The $y$-axis represents values for average illness complexity score and the average natural logarithm for total monthly reimbursements.

The subsequent rows in the table display the mean values of the natural logarithm for average monthly reimbursements in each group, along with standard deviation, minimum, and maximum values.

The average monthly reimbursements for all three methods to evaluate outcome over the study period were not found to demonstrate any significant difference in a one-way ANOVA test. The mean value for the natural logarithm of reimbursement ranged from a low of 4.00 to a high value of 8.65 .

As expected due to the lower sensitivity in CKD staging, 122 patients ended the study year with no change in their CKD stage. This result is contrasted to the distribution of patients according to ending eGFR and ICS. Separation of the pool by ICS demonstrated five patients with an unchanged score from start to end, while 60 improved values and 112

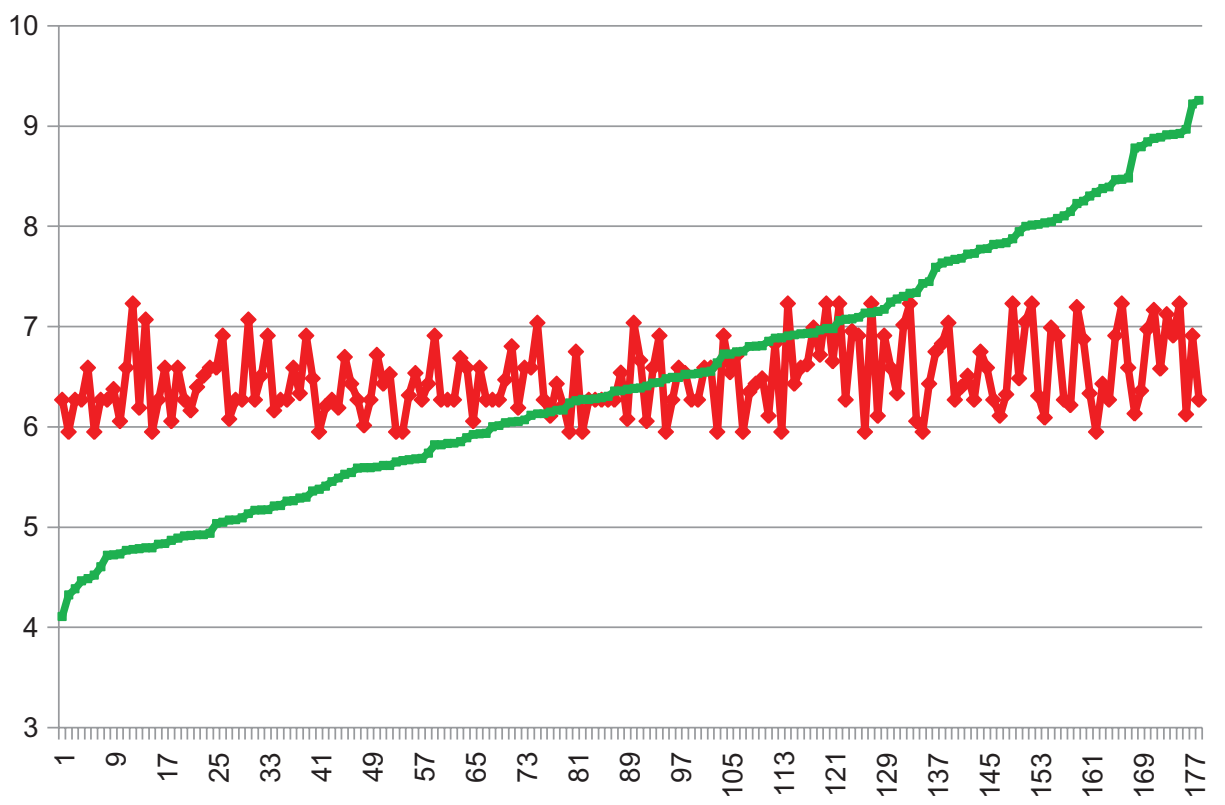

Figure 4 Line graph for 177 renal patients (x-axis) with an average chronic kidney disease stage that ended worse than their starting stage. The $y$-axis represents values for average chronic kidney disease stage and the average natural logarithm for total monthly reimbursements for all delivered medical services. Red line is the complexity score for each patient, while the green line is the average natural logarithm for total monthly payments made for each patient. 
Table 3 Values for the number of patients who completed the study period with the same, better, or worse ending eGFR, ICS, or CKD stage. The remaining rows display the natural logarithm values for mean, minimum, and maximum monthly reimbursement for all delivered health care services, along with group standard deviation

\begin{tabular}{|c|c|c|c|c|c|c|c|c|c|}
\hline & \multicolumn{3}{|c|}{ Ending e-GFR } & \multicolumn{3}{|c|}{ Ending ICS } & \multicolumn{3}{|c|}{ Ending stage } \\
\hline & Same & Better & Worse & Same & Better & Worse & Same & Better & Worse \\
\hline n-pts & 50 & 64 & 63 & 5 & 60 & 112 & 122 & 25 & 30 \\
\hline Mean & 5.53 & 5.70 & 5.80 & 5.29 & 5.64 & 5.85 & 5.71 & 5.95 & 5.84 \\
\hline SD & 1.08 & 1.03 & 1.07 & 1.20 & 0.92 & 1.00 & 0.95 & 1.16 & 0.96 \\
\hline Minimum & 4.71 & 4.00 & 4.07 & 4.28 & 4.21 & 4.00 & 4.00 & 4.21 & 4.59 \\
\hline Maximum & 8.21 & 8.48 & 8.65 & 7.35 & 8.31 & 8.65 & 8.50 & 8.65 & 8.32 \\
\hline
\end{tabular}

Abbreviations: CKD, chronic kidney disease; eGFR, estimated glomerular filtration rate; ICS, illness complexity score; SD, standard deviation; $n$-pts, number of patients.

had a worse ending ICS. This observation is consistent with clinical experience that CKD patients demonstrate progressive debilitating illness, not only in renal function but in associated comorbid conditions. On the other hand, the observed eGFR ending values for the three cohorts demonstrated a distribution of 50,64, and 63 patients within the groups of same, better, and worse, respectively.

The change from start to end eGFR, ICS, and CKD stage in patients with better and worse ending scores were evaluated by a paired $t$-test. Each group demonstrated ending values that were significantly different from starting values at $P$ value $=0.005$. As seen in Table 3 , due to the wide range of reimbursements seen in each group, an ANOVA calculation did not reveal a significant difference in average monthly reimbursements between groups.

An objective of this study was to compare the association of ICS and CKD stage to reimbursement. Since ICS demonstrated a better relationship to reimbursement, a plot of predicted cost vs the ICS linear predictor was calculated. The resultant calculation for predicted average monthly dollars is shown as the plot of the predicted cost vs ICS in Figure 5.

The $\mathrm{x}$-axis demonstrates the linear predictor for each patient and the y-axis is the calculated exponential for each patient's linear predictor in dollars. For linear predictor

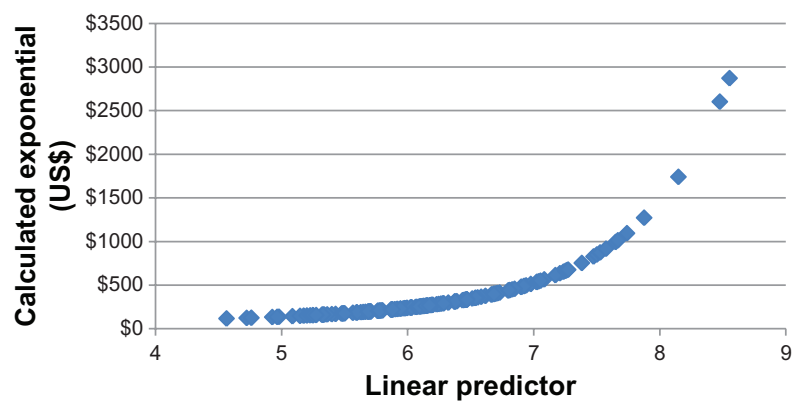

Figure 5 Scatter plot of predicted average monthly cost for the illness complexity score linear predictor in 177 chronic kidney disease patients. The x-axis demonstrates the linear predictor for each patient and the $y$-axis is the calculated exponential for each patient's linear predictor in dollars. values below 7.0, the average monthly reimbursement for these patients is predicted at under US $\$ 500$. As the predictor value increases to 7.5 , the predicted reimbursement doubles to US\$1000 per month. When the predictor reaches a value of 8.65 the predicted monthly reimbursement increases to nearly US\$3000 monthly.

\section{Discussion}

As patients, payers, and elected officials seek to improve the public health and lower health care costs, there is the need to understand the correlation between illness complexity, outcome, and reimbursement. Recent legislation to reform health care and provide universal coverage mandates a shift in provider compensation to a system that rewards valuebased outcomes. Generally, when we consider payment for professional services, intuitively we expect cost to parallel problem complexity, ie, the more severe the problem, the higher the expected cost. Conversely, if the problem is routine, so is the expected fee. Based on this assumption, the goal of this study was to evaluate how routine blood tests commonly ordered by family physicians and nephrologists are related to predicting cost. Since CKD patients typify individuals with a progressive chronic disease, which is complicated by multiple comorbid conditions, such patients were elected for study to evaluate routine blood chemistry profiles that capture indications for liver disease, cardiac disease, anemia, infection, and other conditions associated with CKD. The next objective was to determine if this additional information had a relationship to reimbursement, and if it provided more sensitivity than analysis of claims data and stage of CKD.

In order to accomplish these objectives, a derivative of select blood chemistry values was evaluated to develop an ICS and determine if that numeric value reliably related to reimbursement. Next ICS were examined to determine if it offered more information about disease severity than CKD staging alone. The results of this study demonstrated that 
average ICS values had a reliable association with predicted average monthly reimbursements at an $R^{2}$ value of 0.41 . When that value was compared to the association between average CKD stage and average monthly reimbursement, it revealed an $R^{2}$ value of 0.08 . Thus, ICS offered five times greater sensitivity over CKD staging as a measure of illness complexity.

A major concern for payers, under any system, is that providers will revert to a fee-for-service concept, which incentivizes the use of more procedures. Without reliable objective tools to score illness complexity and outcome, both providers and payers must then depend on anecdotal arguments to debate disagreements over patient complexity and fair reimbursement. The inability to predict expected treatment cost in individual patients presents a challenge to future accountable care organizations as they develop riskadjusted capitation agreements. Although Kidney Disease: Outcomes Quality Initiative/Kidney Disease: Improving Global Outcomes are expected to imminently release an update to CKD staging that employs eGFR and proteinuria/ albuminuria to classify CKD, these authors believe ICS scaling, which accounts for additional comorbid conditions, can augment the sensitivity of upcoming disease specific classifications. In this manner, payers can evaluate claims based on data for individual patients rather than employing global categories based on broad classification of disease by stage. The comorbidities that occur in CKD patients are as, or more, important determinates of illness complexity than kidney function. While it is important to develop improved measures of kidney function, it is also important to develop an illness complexity index that reflects all aspects of complexity and not simply the root cause of that complexity.

This study was undertaken to begin a dialog and suggest further research in developing a dynamic measuring tool that scales illness complexity over the course of treatment in CKD patients, while still respecting the concerns of overutilization in health care services. Such a tool can augment current metrics based on ordinal staging of CKD. The ICS discussed in this study is derived from the summation of a constant (ie, intercept) and beta-weighted values of patient age and select serum chemistry values, which produced a single score based for the standardized deviation of blood tests from their normal mean. The beta-weights (regression coefficients) were calculated from a linear regression of average Z-scores for each blood test for each patient in the study pool on the natural logarithm of average total monthly reimbursements for those same patients. The resultant regression coefficients were then subsequently used to weight the most significant blood test results shown in Table 2 for any patient on any single date of service. The final ICS for any given date of service was based on these weighted factors. With future access to larger data pools, with more longitudinal observations for each variable, the reliability for these coefficients are likely to improve.

These authors understand that staging renal disease by a calculated eGFR is a gold standard for evaluating patients with kidney dysfunction. However, the concern is that determining payment for health care services based primarily on this measure does not illuminate the impact of comorbid conditions or account for different outcomes influenced by this additional complexity. Though there are many other tests which could be employed in a CKD population, this study was restricted to those serum chemistry values considered by the consulting nephrologists to be important in monitoring CKD patients, and importantly were often ordered by primary care physicians as part of a routine blood panel.

The development of disease specific severity scales is not new. Indeed, an example study by Kriegsman et al described how many factors including patient mobility are significantly impacted by complications arising from comorbid conditions that influence a primary chronic disease. ${ }^{18}$ Other authors also analyzed how different chronic diseases had specific characteristics which explained mobility limitations particularly in the elderly. ${ }^{19,20}$ The impact of arthritis, cardiac disease, cerebrovascular disorders, chronic pulmonary disease, and diabetes, many of which accompany CKD can significantly alter a value-based treatment outcome.

However, many articles which report disease specific severity indexes on outcome, base their improvements on refinements achieved through better classification of claims data such as diagnosis-related groups, International Statistical Classification of Diseases and Related Health Problems-9, or discharge summary reports. ${ }^{21-24}$ These authors believe ICS based on the association of select blood chemistry values to reimbursement adds a level of dynamic objectivity to changing health status in chronically ill patients, and potentially offers synergy to current methods of grading based on stage classification.

\section{Study limitations}

Due to the need for specific and repeated blood chemistry tests, this preliminary study of 177 CKD patients was distilled from a renal registry containing 888 patients. With the ever increasing use of electronic health records, along with the availability of physical measurements, such as systolic blood pressure, body mass index, microalbuminuria, and 
cardiac function studies, which could be added to the linear predictors employed in this study, these authors believe the relationship between ICS and reimbursement can be further improved.

In dealing with a small data set, the question of comparison to a validation data set arises. In addition, since the study requirements included the repeated collection of all selected blood tests, the test population could be influenced by selection bias. These individuals could be sicker, or different in some important way from the larger population. These authors believe that is not the case in our study since several parameters within the test group were compared to the larger population of 888 CKD patients who had the required blood tests but not on the repeated protocol which we required. In regards to age, average stage of CKD, and average eGFR, the smaller test group had values of 67 years, stage 3.6 , and $30.5 \mathrm{~mL} /$ minute, while the larger group had 68 years, stage 3.6 , and $35.2 \mathrm{~mL} /$ minute. The average $\mathrm{Z}$-scores for serum creatinine and hemoglobin values in the smaller test group were 8.1 and -2.3 , while the values in the larger group were 6.0 and -2.2 , respectively. In terms of reimbursements, the test group had average monthly costs of US\$1426 while the larger group had US\$1696.

\section{Summary}

In summary, it was found that:

- An ICS derived as the predicted value of the average logarithm of reimbursement from the linear regression on patient age and several select serum chemistry values in CKD patients produces a single score that significantly relates reimbursement to illness complexity $\left(R^{2}=0.41\right.$, $P=0.0005)$.

- Sorting the same patient population by CKD stage and relating it through a simple linear regression to the natural logarithm of average monthly reimbursement demonstrated an $R^{2}$ value of only 0.08 . Thus ICS demonstrated sensitivity five times greater.

- Sorting the patient population by change in CKD stage, ICS, and eGFR over the entire study period demonstrated significant differences in starting and ending values. When the study pool was sorted by CKD stage 122 of 177 patients ended the period with the same stage as they began. On the other hand, sorting of the pool by ICS revealed that 112 of 177 patients ended the period with worse scores than at start. Sorting of the pool by eGFR revealed a nearly even distribution of patients with same, better or worse ending eGFRs.

- Since ICS demonstrated a better relationship to average monthly reimbursement, the exponential linear predictor for ICS was plotted as predicted monthly cost for CKD patients with increasing illness severity.

\section{Disclosure}

The authors report no conflicts of interest in this work.

\section{References}

1. Chassin MR, Galvin RW. The urgent need to improve health care quality: Institute of Medicine National Roundtable on Health Care Quality. JAMA. 1998;280(11):1000-1005.

2. Institute of Medicine. America's health in transition: protecting and improving quality. Washington, DC: National Academy Press; 1994.

3. Petersen LA, Woodard LD, Urech T, Daw C, Sookanan S. Does pay-for-performance improve the quality of health care? Ann Intern Med. 2006;145(4):265-272.

4. Shen Y. Selection incentives in a performance-based contracting system. Health Serv Res. 2003;38(2):535-552.

5. Norton EC. Incentive regulation of nursing homes. $J$ Health Econ. 1992;11(2):105-128.

6. Rosenthal MB, Frank RG, Li Z, Epstein AM. Early experience with pay-for-performance: from concept to practice. JAMA. 2005;294(14):1788-1793.

7. Porter ME, Teisberg EO. Redefining health care: creating value-based competition on results. Boston, MA: Harvard Business School Press; 2006.

8. Baker LC. Measuring competition in health care markets. Health Serv Res. 2001;36(1 Pt 2):223-251.

9. Scanlon DP, Swaminathan S, Lee W, Chernew M. Does competition improve health care quality? Health Serv Res. 2008;43(6): 1931-1951.

10. Landon BE, Zaslavsky AM, Beaulieu ND, Shaul JA, Cleary PD. Health plan characteristics and consumers' assessments of quality. Health Aff (Millwood). 2001;20(2):274-286.

11. Scanlon DP, Darby C, Rolph E, Doty HE. The role of performance measures for improving quality in managed care organizations. Health Serv Res. 2001;36(3):619-641.

12. Johnson CA, Levey AS, Coresh J, Levin A, Lau J, Eknoyan G. Clinical practice guidelines for chronic kidney disease in adults: part 1. Definition, disease stages, evaluation, treatment, and risk factors Am Fam Physician. 2004;70(5):869-876.

13. Smith DH, Gullion CM, Nichols G, Keith DS, Brown JB. Cost of medical care for chronic kidney disease and comorbidity among enrollees in a large HMO population. J Am Soc Nephrol. 2004;15(5):1300-1306.

14. Born PH, Simon CJ. Patients and profits: the relationship between HMO financial performance and quality of care. Health Aff (Millwood). 2001;20(2):167-174.

15. Kessler DP, Geppert JJ. The effects of competition on variation in the quality and cost of medical care. J Econ Manage Strategy. 2005;14(3):575-589.

16. McGlynn EA, Asch SM, Adams J, et al. The quality of health care delivered to adults in the United States. $N$ Engl $J$ Med. 2003;348(26):2635-2645.

17. Bessette R, Carter R. Predicting hospital cost in CKD patients through blood chemistry values. BMC Nephrol. 2011. In press.

18. Kriegsman DM, Deeg DJ, van Eijk JT, Phennix BW, Boeke AJ. Do disease specific characteristics add to the explanation of mobility limitations in patients with different chronic diseases? A study in the Netherlands. J Epidemiol Community Health. 1997;51(6):676-685.

19. Van Den Bos GAM. The burden of chronic diseases in terms of disability, use of health care and healthy life expectancies. Eur J Public Health. 1995;5(1):29-34.

20. Asenjo MA, Bare L, Bayas JM, et al. Relationship between severity, costs and claims of hospitalized patients using the severity of illness index. Eur J Epidemiol. 1994;10(5):625-632. 
21. Freeman JL, Fetter RB, Park H, et al. Diagnosis-related group refinement with diagnosis- and procedure-specific comorbidities and complications. Med Care. 1995;33(8):806-827.

22. Muldoon JH. Structure and performance of different DRG classification systems for neonatal medicine. Pediatrics. 1999;103(1 Suppl E): 302-318.
23. Edwards N, Honemann D, Burley D, Navarro M. Refinement of the Medicare diagnosis related groups to incorporate a measure of severity. Health Care Financ Rev. 1994;16(2):45-64.

24. Leary RS, Johantgen ME, Farley D, Forthman MT, Wooster LD. All-payer severity adjusted diagnosis related groups: a uniform method to severity adjust discharge data. Top Health Inf Manage. 1997;17(3):60-71.

\section{Publish your work in this journal}

The International Journal of Nephrology and Renovascular Disease is an international, peer-reviewed open-access journal focusing on the pathophysiology of the kidney and vascular supply. Epidemiology, screening, diagnosis, and treatment interventions are covered as well as basic science, biochemical and immunological studies. The journal welcomes original research, clinical studies, reviews \& evaluations, expert opinion and commentary, case reports and extended reports. The manuscript management system is completely online and includes a very quick and fair peerreview system, which is all easy to use. Visit http://www.dovepress.com/ testimonials.php to read real quotes from published authors.

Submit your manuscript here: http://www.dovepress.com/international-journal-of-nephrology-and-renovascular-disease-journal 